\title{
Clinical Outcomes and Costs of Rivaroxaban for Thromboprophylaxis in Acutely Ill Medical Inpatients: A Cross-Sectional Study
}

Gustavo Lenci Marques ${ }^{1}$, Ana Carolina De Franca ${ }^{1}$, Ana Carolina Saito ${ }^{1}$, Fabiana L. Hornung ${ }^{1}$, Ana Carolina Motter ${ }^{1}$, Ana Carolina Falzoni Pontello ${ }^{1}$, Helena Fontana ${ }^{1}$, Juliano Gasparetto ${ }^{1}$, Tiago Zequinão 1

1. Internal Medicine, Pontifical Catholic University of Parana, Curitiba, BRA

Corresponding author: Gustavo Lenci Marques, gustavolencimarques@gmail.com

\begin{abstract}
Introduction: Venous thromboembolism (VTE) is the primary cause of preventable death in hospitalized patients in the United States. This is a cross-sectional study with a brief cost analysis of thromboprophylaxis with rivaroxaban and enoxaparin in acutely ill medical inpatients.

Methods: The study included a total of 122 patients admitted to a public teaching hospital from December 2019 to January 2021. The sample was equally divided into two groups according to the thromboprophylactic agent prescribed: rivaroxaban or enoxaparin. The primary outcomes included bleeding and symptomatic, ultrasonography-confirmed arterial or venous thrombotic events during or within 90 days after hospitalization. Our secondary outcome was the direct costs of each anticoagulant in US dollars over the 14 months.
\end{abstract}

Results: During hospitalization, two events were detected in the enoxaparin group: minor bleeding with minimum intervention required (1.6\%) and a deep vein thrombosis (DVT) case (1.6\%) confirmed by ultrasonography. Within 90 days after discharge, two patients, one of each sample (1.6\% vs. $1.6 \%)$, were readmitted due to confirmed acute arterial occlusion. Concerning financial assessment, the mean unit cost of enoxaparin during the 14 months assessed was $102.14 \%$ more expensive than rivaroxaban.

Conclusions: Both rivaroxaban and enoxaparin showed equivalence in effectiveness and safety in thromboprophylaxis in medical inpatients, aside from possible financial benefit with the first-mentioned drug.

Categories: Internal Medicine

Keywords: thromboprophylaxis, acutely ill medical inpatients, cost assessment, rivaroxaban, enoxaparin

Review began 05/08/2021 Review ended 05/26/2021 Published 06/07/2021

๑) Copyright 2021 Lenci Marques et al. This is an open access article distributed under the terms of the Creative Commons Attribution License CC-BY 4.0., which permits unrestricted use, distribution, and reproduction in any medium, provided the original author and source are credited.

\section{Introduction}

Venous thromboembolism (VTE), a common medical condition worldwide, is the primary cause of preventable death in hospitalized patients in the United States and has an estimated mortality rate ranging from $10 \%$ to $30 \%$ within 30 days [1-2]. Hospitalization due to acute medical illness, major surgery, prolonged immobilization, active cancer, and advanced age ( $\geqslant 75$ years) are known risk factors for deep vein thrombosis (DVT) and pulmonary embolism (PE) [3-4]. Current drugs used for thromboprophylaxis in acutely ill medical inpatients include low-molecular-weight heparin (LMWH), unfractionated heparin (UFH), and fondaparinux, as recommended by the American College of Chest Physicians (ACCP) guidelines [5]. However, some disadvantages of these medications, such as parenteral administration and erratic absorption, have encouraged new studies on alternative anticoagulants for thromboprophylaxis in the aforementioned population [6]. Moreover, several studies have shown that other agents, such as rivaroxaban, can be more cost-effective than LMWH in thromboprophylaxis [7].

Rivaroxaban is an oral direct factor Xa inhibitor widely used in the prevention of VTE after major orthopedic surgery. It has been proven to be an effective and safe thromboprophylactic drug when compared to enoxaparin in patients submitted to hip and knee arthroplasty, as well as after below-knee lower-leg fracture surgery [8-11]. Regarding thromboprophylaxis in medical inpatients, the MAGELLAN trial reported noninferiority of rivaroxaban effectiveness and safety when compared to enoxaparin [4].

Until recently, rivaroxaban was used as a thromboprophylactic agent for orthopedic patients only. In 2019, however, this anticoagulant was also approved by the Food and Drug Administration (FDA) for thromboprophylaxis in acutely ill medical patients, even though it is not widely used in these scenarios yet [12-13]. Therefore, our objective in the current study is to evaluate whether rivaroxaban was as safe and effective as enoxaparin in the prevention of VTE in acutely ill medical inpatients. Major risk factors for thrombosis have also been considered. Furthermore, we compared the direct costs of thromboprophylaxis 
with each anticoagulant.

\section{Materials And Methods \\ Study design and setting}

This is a cross-sectional study with a brief cost analysis of thromboprophylaxis with rivaroxaban and enoxaparin in acutely ill medical inpatients. This study was conducted at Cajuru University Hospital, a 206bed public teaching hospital specialized in medical and surgical emergencies in the city of Curitiba, Brazil. A total of 122 patients admitted to the internal medicine service from December 2019 to January 2021 were included, equally divided into two groups between oral rivaroxaban, $10 \mathrm{mg}$ daily, and subcutaneous enoxaparin, $40 \mathrm{mg}$ daily. This study was approved by the local ethics committee (Comitê de Ética em Pesquisa da Pontifícia Universidade Católica do Paraná) under approval number 38454620500000020. Informed consent from participants was waived by the Institutional Review Board.

\section{Eligibility criteria}

Patients included were above 18 years, who completed anticoagulation with rivaroxaban or enoxaparin for at least two days during hospitalization for acute medical illness, except when DVT/PE was suspected. Patients with a history of continuous anticoagulation therapy or dual antiplatelet therapy were excluded, as well as patients who required intensive care or who underwent surgical procedures due to the primary reason for hospitalization. Patients with active leukemia or metastatic cancer were also excluded. The sample size was determined by the number of patients who used rivaroxaban until the deadline of the study.

\section{Assessment and outcomes}

Comorbidities were considered according to the Charlson Comorbidity Index, whereas risk factors for VTE followed those considered in the MAGELLAN trial, which included age $\geqslant 75$ years, acute infectious disease, history of VTE, hormone-replacement therapy, major surgery, or serious trauma within the previous 6-12 weeks and hereditary or acquired thrombophilia. We did not consider body mass index. The primary outcomes included bleeding (classified as minimum, minor, major, or fatal) and symptomatic, ultrasonography-confirmed arterial or venous thrombotic event during or within 90 days after hospitalization. Our secondary outcome was the direct costs of each anticoagulant in US dollars over the 14 months.

\section{Cost analysis}

Initially, expenses with each medication were extracted from the hospital pharmacy spreadsheets in the local currency (Brazilian Reals). The average monthly costs of each drug during the period analyzed were calculated and then converted to US dollars according to its average value provided by the Central Bank of Brazil throughout the same period. The average US dollar value corresponded to BRL 5.09, which led us to the average costs of rivaroxaban and enoxaparin per unit. Afterward, these values were multiplied by total days of anticoagulation and the direct mean costs of each thromboprophylactic drug were obtained.

\section{Statistical analysis}

Descriptive statistical analysis was performed for all numeric variables. We performed a Student's t-test or Mann-Whitney test to compare numerical data. For dichotomous data, Fisher's exact test was used to compare proportions between the two prophylactic agents. All data were tabulated in Microsoft Excel spreadsheets and statistical analysis was performed using Statistical Package for Social Science 21.0 (SPSS 21.0, IBM Corp., Armonk, NY, USA).

\section{Results}

\section{Participants}

From December 2019 to January 2021, 824 patients admitted to the internal medicine service used enoxaparin or rivaroxaban for thromboprophylaxis during their admission. A total of 151 patients from the initial sample used rivaroxaban, out of which 61 were eligible. Due to the limited number of patients undergoing prophylaxis with rivaroxaban, only 256 medical records were evaluated from the enoxaparin group, resulting in equal samples, each with 61 patients (Figure 1). Among the analyzed sample, the majority of patients were male (62.3\%), with an average age of 60.2 years and a mean hospitalization length of 9.9 days. Other characteristics of the sample included a 1.37 average Charlson Comorbidity Index, prophylaxis extent of 6.9 days, and a total of six deaths in 30 days, from any cause. The main causes of hospitalization were: infectious disease (44.3\%); non-surgical trauma (15.6\%); abdominal condition (9.0\%); metabolic disorder, including electrolyte disturbances, hypoglycemia, wasting syndrome and hypoalbuminemia (7.4\%); cardiovascular disease (5.7\%); neurological disorder (5.7\%); intoxication (4.1\%); respiratory disease (4.1\%); urinary tract disorder (2.5\%); and inflammatory or rheumatic disease (1.6\%). Comorbidities and risk factors were described in Table 1. 


\section{Cureus}

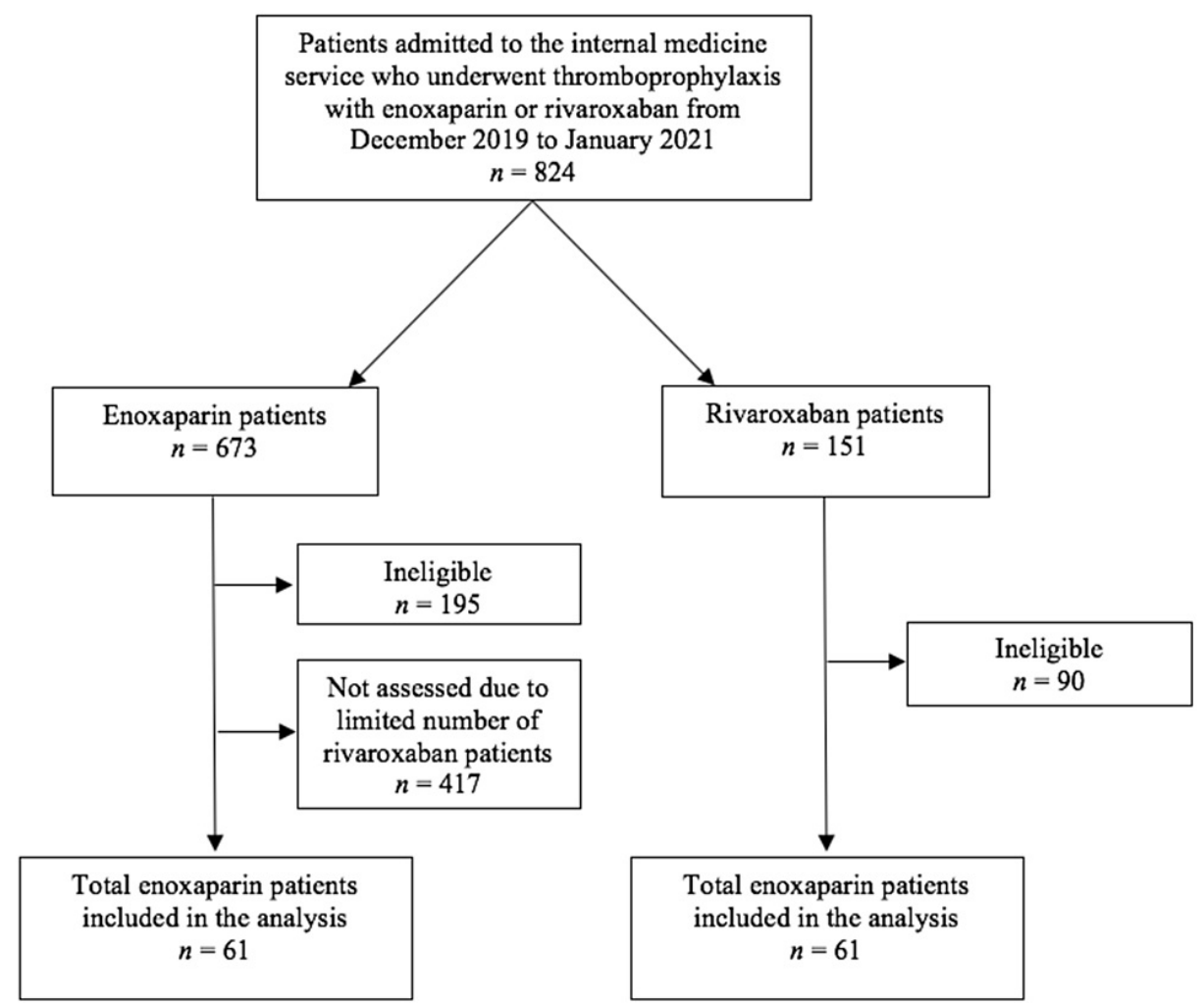

FIGURE 1: Flow diagram of sample selection.

\begin{tabular}{|c|c|c|}
\hline Characteristic & Enoxaparin $(\mathrm{N}=61)$ & Rivaroxaban $(\mathrm{N}=61)$ \\
\hline Mean age (SD) - yr & $61.7(16.9)$ & $58.6(20.7)$ \\
\hline Male sex - no * & $32(52.5 \%)$ & $44(72.1 \%)$ \\
\hline Median duration of hospitalization (IQR) - days & $8(5-13.5)$ & $7(5-11.5)$ \\
\hline Median Charlson Comorbidity Index (IQR) & $1(0-2)$ & $1(0-2)$ \\
\hline Median duration of anticoagulation (IQR) - days * & $6(4-10)$ & $4(3-7.5)$ \\
\hline Comorbidity - no & - & - \\
\hline History of MI & $6(9.8 \%)$ & $3(4.9 \%)$ \\
\hline Congestive heart failure & $4(6.6 \%)$ & $4(6.6 \%)$ \\
\hline Peripheral vascular disease & $1(1.6 \%)$ & $3(4.9 \%)$ \\
\hline Cerebrovascular disease & $7(11.5 \%)$ & $6(9.8 \%)$ \\
\hline Dementia & $11(18 \%)$ & $6(9.8 \%)$ \\
\hline Chronic pulmonary disease & $5(8.2 \%)$ & $3(4.9 \%)$ \\
\hline Rheumatic disease * & $6(9.8 \%)$ & 0 \\
\hline Peptic ulcer disease & $2(3.3 \%)$ & $1(1.6 \%)$ \\
\hline Mild liver disease & $4(6.6 \%)$ & $2(3.3 \%)$ \\
\hline Diabetes without end-organ damage & $12(19.7 \%)$ & $11(18.0 \%)$ \\
\hline Diabetes with end-organ damage & $4(6.6 \%)$ & $3(4.9 \%)$ \\
\hline Moderate to severe renal disease & 0 & 0 \\
\hline Hemiplegia & 0 & 0 \\
\hline
\end{tabular}




\section{Cureus}

\begin{tabular}{|c|c|c|}
\hline Any malignancy without metastases & $3(4.9 \%)$ & $5(8.2 \%)$ \\
\hline Leukemia & 0 & 0 \\
\hline Lymphoma & 0 & 0 \\
\hline Metastatic tumor & 0 & 0 \\
\hline Moderate or severe liver disease & 0 & 0 \\
\hline AIDS & $3(4.9 \%)$ & $2(3.3 \%)$ \\
\hline Risk factor for DVT - no & - & - \\
\hline Age $\geq 75$ years & $13(21.3 \%)$ & $13(21.3 \%)$ \\
\hline History of cancer & $3(4.9 \%)$ & $5(8.2 \%)$ \\
\hline History of heart failure $\dagger$ & $4(6.6 \%)$ & $4(6.6 \%)$ \\
\hline Acute ischemic stroke with leg paresis & $6(9.8 \%)$ & $6(9.8 \%)$ \\
\hline Acute infectious disease & $30(49.2 \%)$ & $37(60.7 \%)$ \\
\hline Severe varicosis & $1(1.6 \%)$ & $1(1.6 \%)$ \\
\hline History of DVT or PE & 0 & $2(3.3 \%)$ \\
\hline Hormone-replacement therapy & $2(3.3 \%)$ & $2(3.3 \%)$ \\
\hline Major surgery within the previous $6-12$ weeks & $3(4.9 \%)$ & $8(13.1 \%)$ \\
\hline Serious trauma within the previous $6-12$ weeks & $10(16.7 \%)$ & $9(14.8 \%)$ \\
\hline Hereditary or acquired thrombophilia & 0 & 0 \\
\hline Chronic venous insufficiency & $2(3.3 \%)$ & $2(3.3 \%)$ \\
\hline Death within 30 days - no & $4(6.6 \%)$ & $2(3.3 \%)$ \\
\hline Acute medical condition - no & - & - \\
\hline Abdominal condition & $5(8.2 \%)$ & $6(9.8 \%)$ \\
\hline Cardiovascular disease & $3(4.9 \%)$ & $4(6.6 \%)$ \\
\hline Infectious disease & $28(45.9 \%)$ & $26(42.6 \%)$ \\
\hline Inflammatory or rheumatic disease & $1(1.6 \%)$ & $1(1.6 \%)$ \\
\hline Intoxication & $3(4.9 \%)$ & $2(3.3 \%)$ \\
\hline Metabolic disorder $\ddagger$ & $4(6.6 \%)$ & $5(8.2 \%)$ \\
\hline Neurological disorder & $4(6.6 \%)$ & $3(4.9 \%)$ \\
\hline Non-surgical trauma & 7 (11.5\%) & 12 (19.7\%) \\
\hline Respiratory disease & $4(6.6 \%)$ & $1(1.6 \%)$ \\
\hline Urinary tract disorder & $2(3.3 \%)$ & $1(1.6 \%)$ \\
\hline
\end{tabular}

\section{TABLE 1: Baseline characteristics of patients.}

SD, standard deviation; IQR, interquartile range; MI, myocardial infarction; AIDS, acquired immunodeficiency syndrome; DVT, deep vein thrombosis; $\mathrm{PE}$, pulmonary embolism

${ }^{*} p$ values $\leq 0.05$

†Heart failure patients included were New York Heart Association class III or IV

‡Metabolic disorder included electrolyte disturbances, hypoglycemia, wasting syndrome, and hypoalbuminemia

\section{Outcome data}


Throughout the analyzed period, two events were detected in the enoxaparin group: minor bleeding with minimum intervention required (1.6\%) and a DVT case (1.6\%) confirmed by ultrasonography. No primary outcome event was registered in the rivaroxaban group during hospitalization. Within 90 days after discharge, two patients, one of each sample (1.6\% vs. 1.6\%), were readmitted due to confirmed acute arterial occlusion. No significant difference was seen with respect to primary outcomes, as described in Table 2.

\begin{tabular}{|l|c|}
\hline Characteristic & Enoxaparin \\
\hline DVT during hospitalization & $1(1.6 \%)$ \\
\hline Bleeding during hospitalization & $1(1.6 \%)$ \\
\hline Thrombotic event within 90 days after hospitalization & $1(1.6 \%)$ \\
\hline
\end{tabular}

TABLE 2: Primary outcomes.

$p$ values $>0.05$

DVT, deep vein thrombosis

Our secondary outcome was to assess the direct mean costs of each medication. Considering the average USD value corresponding to BRL 5.09, the mean unit cost of rivaroxaban was USD 1.40, while the mean unit cost of enoxaparin was USD 2.83. The total expenditure after 14 months in the rivaroxaban group was approximately USD 464.80, which contrasts with a total expenditure of approximately USD 1,443.30 in the enoxaparin group.

\section{Discussion}

The present study assessed the effectiveness and safety of thromboprophylaxis in hospitalized medical patients submitted to anticoagulation with rivaroxaban or enoxaparin. Although limited research regarding thromboprophylaxis has been performed on acutely ill inpatients, our results were similar to those reported in the literature. During an average of 6.9 days of thromboprophylaxis, only minor bleeding and a DVT case were accounted for in the enoxaparin group, whereas no events were observed in the rivaroxaban group. Within the following 90 days, two arterial thrombotic events were reported, one in each group.

Regardless of a similar risk factor prevalence in both samples, more cases of thrombosis and bleeding were reported in the enoxaparin group, but the statistical difference was not significant. The higher incidence of thrombotic events in the enoxaparin group was also described in orthopedic studies, suggesting rivaroxaban may be more effective in thromboprophylaxis [10-11]. As opposed to results found in previous research, however, rivaroxaban was not associated with higher bleeding rates [4].

As a secondary outcome, the study analyzed the direct costs of each drug. The mean unit cost of enoxaparin during the 14 months assessed was $102.14 \%$ more expensive than rivaroxaban. What this means is that, throughout the same period, thromboprophylaxis with rivaroxaban could have spared USD 978.50. These findings are consistent with a cost-effectiveness study on orthopedic patients that also showed rivaroxaban could be less costly than LMWH [7]. Indirect costs, such as administration and disposal time by the nurse staff, are hypothesized to be higher with enoxaparin thromboprophylaxis, but further studies are required for confirmation. The possibility of thromboprophylaxis for a much lower cost is an important issue, particularly for developing countries, such as Brazil, in which a public national health system takes place.

Our study has some limitations, such as the inherent limitation of the retrospective study design, the size of the sample (restricted by the number of patients in the use of rivaroxaban throughout the analyzed period), and the inclusion of only one hospital. Regarding data collection, patient information was limited to medical records. Therefore, if not specified, it was assumed that patients did not present any additional conditions or take any additional medications. Concerning bleeding assessment, risk factors such as active gastroduodenal ulcer, prior bleeding, low platelet count, hepatic or renal failure, and the presence of a central venous catheter were not considered [14]. Moreover, in accordance with the hospital recommendations, ultrasonography to confirm thrombotic events was performed only in symptomatic patients, which may have underestimated VTE or arterial occlusion outcomes.

Despite the described limitations, the study included a population with broad characteristics, comprising a wide age range. Additionally, the differences observed in the prevalence of comorbidities between the groups did not affect statistics in VTE risk. Therefore, the effectiveness and safety of thromboprophylaxis with rivaroxaban were similar to enoxaparin. A final worth mentioning benefit of thromboprophylaxis with rivaroxaban regards its route of administration. The convenience and safety of oral drug administration, 


\section{Conclusions}

Both rivaroxaban, at a dose of $10 \mathrm{mg}$ once daily, and enoxaparin, at a dose of $40 \mathrm{mg}$ once daily, showed equivalence in effectiveness and safety in thromboprophylaxis in medical inpatients, aside from possible financial benefit with the first-mentioned drug. Further randomized controlled clinical trials are required to support our findings. For future research, the inclusion of a broader number of participants is recommended in order to identify thrombotic events on a larger scale.

\section{Additional Information \\ Disclosures}

Human subjects: Consent was obtained or waived by all participants in this study. Comitê de Ética em Pesquisa da Pontifícia Universidade Católica do Paraná issued approval 38454620500000020. This study was approved by the local ethics committee (Comitê de Ética em Pesquisa da Pontifícia Universidade Católica do Paraná) under approval number 38454620500000020 . Informed consent from participants was waived by the Institutional Review Board. Animal subjects: All authors have confirmed that this study did not involve animal subjects or tissue. Conflicts of interest: In compliance with the ICMJE uniform disclosure form, all authors declare the following: Payment/services info: All authors have declared that no financial support was received from any organization for the submitted work. Financial relationships: All authors have declared that they have no financial relationships at present or within the previous three years with any organizations that might have an interest in the submitted work. Other relationships: GM received personal fee from Bayer, Boehringer Ingelheim and Astrazeneca for consulting and speaker bureau. JG is executive director of Cajuru University Hospital.

\section{References}

1. Raskob GE, Angchaisuksiri P, Blanco AN, et al.: Thrombosis: a major contributor to global disease burden . Semin Thromb Hemost. 2014, 40:724-735. 10.1055/s-0034-1390325

2. Beckman MG, Hooper WC, Critchley SE, Ortel TL: Venous thromboembolism: a public health concern. Am J Prev Med. 2010, 38:S495-S501. 10.1016/j.amepre.2009.12.017

3. Wendelboe AM, Raskob GE: Global burden of thrombosis: epidemiologic aspects . Circ Res. 2016, 118:13401347. 10.1161/CIRCRESAHA.115.306841

4. Cohen AT, Spiro TE, Büller HR, et al.: Rivaroxaban for thromboprophylaxis in acutely ill medical patients . N Engl J Med. 2013, 368:513-523. 10.1056/NEJMoa1111096

5. Kahn SR, Lim W, Dunn AS, et al.: Prevention of VTE in nonsurgical patients: antithrombotic therapy and prevention of thrombosis, 9th ed: American College of Chest Physicians Evidence-Based Clinical Practice Guidelines. Chest. 2012, 141:e195S-e226S. 10.1378/chest.11-2296

6. Al Yami MS, Kurdi S, Abraham I: Direct oral anticoagulants for extended thromboprophylaxis in medically ill patients: meta-analysis and risk/benefit assessment. J Blood Med. 2018, 9:25-34. 10.2147/JBM.S149202

7. Mahmoudi M, Sobieraj DM: The cost-effectiveness of oral direct factor Xa inhibitors compared with lowmolecular-weight heparin for the prevention of venous thromboembolism prophylaxis in total hip or knee replacement surgery. Pharmacotherapy. 2013, 33:1333-1340. 10.1002/phar.1269

8. Loganathan V, Hua A, Patel S, Gibbons C, Vizcaychipi MP: Efficacy and safety of rivaroxaban thromboprophylaxis after arthroplasty of the hip or knee: retrospective cohort study. Ann R Coll Surg Engl. 2016, 98:507-515. 10.1308/rcsann.2016.0197

9. Sindali K, Rose B, Soueid H, Jeer P, Saran D, Shrivastava R: Elective hip and knee arthroplasty and the effect of rivaroxaban and enoxaparin thromboprophylaxis on wound healing. Eur J Orthop Surg Traumatol. 2013, 23:481-486. 10.1007/s00590-012-0987-y

10. Liu J, Zhao J, Yan Y, Su J: Effectiveness and safety of rivaroxaban for the prevention of thrombosis following total hip or knee replacement: a systematic review and meta-analysis. Medicine (Baltimore). 2019, 98:e14539. 10.1097/MD.0000000000014539

11. Lassen MR, Haas S, Kreutz R, Mantovani LG, Holberg G, Turpie AG: Rivaroxaban for thromboprophylaxis after fracture-related orthopedic surgery in routine clinical practice. Clin Appl Thromb Hemost. 2016, 22:138-146. 10.1177/1076029615607303

12. FDA: Approves xarelto (rivaroxaban) to help prevent blood clots in acutely ill medical patients . (2019). Accessed: February 17, 2021: https://www.drugs.com/newdrugs/fda-approves-xarelto-rivaroxaban-helpprevent-blood-clots-acutely-ill-medical-patients....

13. Schünemann HJ, Cushman M, Burnett AE, et al.: American Society of Hematology 2018 guidelines for management of venous thromboembolism: prophylaxis for hospitalized and nonhospitalized medical patients. Blood Adv. 2018, 2:3198-3225. 10.1182/bloodadvances.2018022954

14. Decousus H, Tapson VF, Bergmann JF, et al.: Factors at admission associated with bleeding risk in medical patients: findings from the IMPROVE investigators. Chest. 2011, 139:69-79. 10.1378/chest.09-3081 